\title{
Nefropatía del lupus en la infancia
}

\author{
DRS. GALLETTI A*, ROSEMBERG R. **, ALLENDE N. ** SORENSEN $R^{* * * *}$, PUGA $F . * * *$.
}

InTRoducción. El lupus eritematoso sistémico (LES) es una afección que compronete el tejido conectivo de muchos órganos, especialmente el que forma la pared vascular y las diversas cavidades serosas, dando origen a cuadros muy proteiformes (1) (2). En los nińos la enfermedad tiene un mal pronóstico determinado fundamentalmente por la presencia o ausencia del compromiso renal (2) (3) (4).

Hemos querido presentar nuestra experiencia con pacientes cuyo LES ha sido diagnosticado $\mathrm{y}$ controlado en el Hospital Luis Calvo Mackenna, dejando constancia que los avances inmunológicos han permitido la introducción de nuevas técnicas en el estudio de tejidos renales y con ello la posibilidad de un diagnóstico más precoz (5) (6).

Material y MÉTODo. Se analizan seis pacientes con LES diagnosticados entre los años 1969 1971, todos los cuales presentaron compromiso renal de diversa intensidad. El diagnóstico de LES (7) (8) se fundamentó en el compromiso de por lo menos dos sistemas, células del lupus, gamaglobulinas y VHS elevadas; el compromiso renal se pesquisó por presencia de hematuria, edema o hipertensión arterial, alteraciones del sedimento urinario, uremia y clearence de creatinina; a todos salvo un caso se le practicó biopsia renal.

Resultados. Todos nuestros pacientes son del sexo femenino con edades fluctuantes entre $7 \mathrm{y}$ 11 años, siendo el motivo de consulta muy proteiforme (Tabla 1). Entre las manifestaciones extrarenales, destaca la presencia de fiebre, erite-

* Médico Residente Becario. Hospital Luis Calvo Mackenna.

* Departamento Anatomja Patológica. Universidad Católica de Chile.

** Departamento Nefrología. Hospital Luis Calvo Mackenna.

** Sección Inmunopatologia. Instituto Bacteriológico.

Unidad Inmunología. Hospital Luis Calvo Mackenna,
T A B L A N $^{\circ} 1$

EDAD, SEXO Y MOTIVO DE CONSULTA EN 6 PACIENTES CON LUPUS $Y$ NEFROPATIA

\begin{tabular}{c|c|c|l}
\hline Caso No & Edad & Sexo & Motivo consulta \\
\hline 1 & $10 \mathrm{a}$ & $\mathbf{F}$ & Artritis \\
2 & 10 & F & Eritema \\
3 & 7 & F & Hepatitis \\
4 & 9 & F & Anemia \\
5 & 11 & F & Artritis \\
6 & 10 & F & Sind. Nefrítico \\
\hline
\end{tabular}

ma y artritis en todos ellos (Tabla 2), la leucopenia y pancitopenia fueron hallazgo frecuentes (Tabla 3).

Casos clínicos. Caso 1: corresponde M. M. (156446), fue considerada y tratada como artritis reumatoidea hasta el año cuatro meses de evolución en que se diagnostica LES. Es importante destacar que a los dos meses de evolución de manifestaciones articulares se pesquisó, gracias al se-

T A B L A No 2

SIGNOS CLINICOS EXTRARRENALES EN 6 PACIENTES CON NEFROPATIA LUPICA

\begin{tabular}{l|c}
\hline \multicolumn{1}{c|}{ Signos } & No Casos \\
\hline Fiebre & 6 \\
Eritema & 6 \\
A.rtritis & 6 \\
Poliserositis & 2 \\
Convulsiones & 2 \\
Hepatomegalia & 2 \\
Hepat. y Esplen. & 1 \\
Atrofia Papilar & 1 \\
\hline
\end{tabular}


TABLA No 3

SIGNOS DE LABORATORIO EXTRARRENALES EN 6 PACIENTES CON NEFROPATIA LUPICA

\begin{tabular}{l|c}
\hline \multicolumn{1}{c|}{ Signos } & No Casos \\
\hline V. H. S. $\mathbf{~}$ & $\mathbf{6}$ \\
C. Lúpus & 6 \\
inn. rel alb/gl. & 6 \\
Anemia & 6 \\
Leucoperia & 5 \\
Pancitopenia & 4 \\
\hline
\end{tabular}

dimento urinario, un leve compromiso renal que no se manifestaba clínicamente (Tabla 4). La biopsia renal realizada cuando se diagnosticó LES, reveló una glomerulonefritis difusa, escaso fibrinoide en los glomérulos e inflamación instersticial focal (tabla 5). En la actualidad lleva cinco años dos meses en control en nuestro servicio con buena respuesta al tratamiento (tabla 6).

Caso 2: R. R. (184614) a los cinco meses de sus primeras manifestaciones clínicas se pesquisó compromiso renal (tabla 4). La biopsia reve- ló una glomerulitis predominantemente difusa (tabla 5); ha completado dos años un mes de control con resultados satisfactorios (tabla 6).

Caso 3: Z. M. (88948) estuvo rotulada y tratada como hepatitis prolongada durante dos años tres meses, no habiendo, durante este lapso, alteraciones clínicas o uroanálisis que hicieran sospechar compromiso renal, el cual se manifestó tras un año de alejarse de nuestro control con severas alteraciones en el sedimento y función renal (tabla 4), la biopsia renal reveló glomerulitis difusa leve (tabla 5). La terapia esteroidal logró controlar la afección durante dos años seis meses falleciendo en insuficiencia renal crónica (tabla 6).

Caso 4: I. M. (116452) a los dos meses de iniciada la afección hay evidencias clínicas confirmadas con sedimento urinario y estudios funcionales de un grave compromiso renal (tabla 4). La biopsia reveló una glomerulonefritis difusa con exudación polimorfonuclear con proliferación epitelíal glomérulo capsular abundante, fibrinoide e inflamación instersticial acentuada (tabla 5). Hubo mala respuesta a la prednisona con intensas manifestaciones secundarias que obligaron al uso de metotrexato que le produce una

TA B L A No 4

INTERVALO ENTRE INICIACION LED Y NEFROPATIA, MANIFESTACIONES RENALES INICIALES, TIEMPO EN CONTROL, ESTADO ACTUAL

\begin{tabular}{|c|c|c|c|c|c|c|c|c|c|}
\hline \multirow{2}{*}{$\begin{array}{c}\text { Caso } \\
\text { No }\end{array}$} & \multirow{2}{*}{$\begin{array}{c}\text { Intervalo entire } \\
\text { iniciación } L E D \\
\text { y nefropatía }\end{array}$} & \multicolumn{6}{|c|}{ Manifestaciones renales iniciales } & \multirow{2}{*}{$\begin{array}{c}\text { Tiempo } \\
\text { ent } \\
\text { control }\end{array}$} & \multirow{2}{*}{$\begin{array}{c}\text { Estado } \\
\text { actual }\end{array}$} \\
\hline & & Edcma & Hematuria & $\begin{array}{c}\text { Presión } \\
\text { arterial }\end{array}$ & Proteinuria & Uremia & $\begin{array}{l}\text { Clea- } \\
\text { rence }\end{array}$ & & \\
\hline 1 & 2 meses & - & + & $\mathrm{N}$ & + & N. & N. & 5 a $2 \mathrm{~ms}$. & $\underset{\text { Control }}{\text { En }}$ \\
\hline 2 & 5 meses & + & +++ & N. & + & N. & N. & $2 \mathrm{a} 1 \mathrm{~m}$ & $\begin{array}{c}\text { En } \\
\text { Control }\end{array}$ \\
\hline 3 & 2 años 3 meses & + & +++ & N. & + & Elevada & $\begin{array}{l}\text { No se } \\
\text { hizo }\end{array}$ & 2 a $6 \mathrm{~ms}$. & Fallecido \\
\hline 4 & 2 meses & + & +++ & Elevada & +++ & Elevada & Bajo & 3 ms. & Fállecido \\
\hline 5 & & - & + & N. & + & N. & N. & $3 \mathrm{~ms}$. & Fallecido \\
\hline 6 & & + & +++ & Elevada & +++ & Elevada & $\mathrm{N}$. & $4 \mathrm{~ms}$. & Fallecido \\
\hline
\end{tabular}

T A B L A No 5

HALLAZGOS EN LA BIOPSIA RENAL DE 5 CASOS DE LED. CON NEFROPATIA

\begin{tabular}{|c|c|c|c|c|c|}
\hline Caso & $\begin{array}{c}\text { Eugr. cáps. } \\
\text { Bowman }\end{array}$ & $\begin{array}{c}\text { Necrosis } \\
\text { fibrinoide }\end{array}$ & $\begin{array}{l}\text { Wire } \\
\text { Loop }\end{array}$ & Células & $\begin{array}{c}\text { Hematoxi- } \\
\text { linicos }\end{array}$ \\
\hline 1 & $t+$ & ++ & + & Redondas & + \\
\hline 2 & ++ & - & 一 & Redondas & - \\
\hline 3 & ++ & - & - & - & - \\
\hline 4 & +++ & ++ & + & $\begin{array}{l}\text { Linf. } \\
\text { Plasm. }\end{array}$ & + \\
\hline 5 & +++ & ++ & + & $\begin{array}{l}\text { Linf. } \\
\text { Plasm. }\end{array}$ & - \\
\hline
\end{tabular}


TABLA No 6

MANIFESTACIONES DE NEPROPATIA LUPICA RESPUESTA AL TRATAMIENTO CON PREDNISONA, TIEMPO EN CONTROL. ESTADO ACTUAL Y CAUSA DE MUERTE

\begin{tabular}{|c|c|c|c|c|c|c|}
\hline Caso & Edema & Hematuria & Proteinuria & Uremia & $\begin{array}{l}\text { Tiempo } \\
\text { control }\end{array}$ & $\begin{array}{c}\text { Causa } \\
\text { muerte }\end{array}$ \\
\hline 1 & 0 & 0 & $\div$ & 0 & 5 a $2 \mathrm{~ms}$. & - \\
\hline 2 & + & + & $\dot{T}$ & 0 & 2 a $1 \mathrm{~ms}$. & - \\
\hline 3 & 0 & + & + & + & 2 a $6 \mathrm{~ms}$. & IRCr. \\
\hline 4 & - & - & & & & Aplasia \\
\hline & & & 一 & - & $3 \mathrm{~ms}$. & $\begin{array}{l}\text { medular } \\
\text { iatrogénica }\end{array}$ \\
\hline 5 & - & - & - & - & $3 \mathrm{~ms}$ & Neumonia \\
\hline 6 & 一 & - & - & - & $4 \mathrm{~ms}$. & 1. R. A. \\
\hline
\end{tabular}

$(+)=$ buena respuesta $(-)=\sin$ respuesta $0=$ ausencia.

aplasia medular con fatales resultados a los tres meses de pesquisado el compromiso renal (tabla 6).

Caso 5: C. P. (213602) desde sus primeras manifestaciones de LES presentó un sedimento urinario alterado (tabla 4). No se efectuó biopsia, no se obtuvo respuesta a terapia con esteroides (tabla 6) en el curso de la cual contrae una neumonia que causa su muerte a los tres meses de diagnosticada su enfermedad.

Caso 6: E. P. (21951) el LES se inició con grave compromiso renal (tabla 4) con lesiones histologicas de glomerulonefritis difusa con extensas proliferaciones epiteliales glomérulo capsulares (tabla 5), no hubo respuesta a los esterojdes falleciendo a los cuatro meses de iniciada su sintomatología (tabla 6).

Características histológicas. (Tabla 5) La glomérulonefritis denominada por nosotros característica es aquella que presenta asincronia en la evolución de la inflamación glomerular y la presencia, generalmente en forma segmentaria, de substancia fibrinoide mesangial y subendotelial a veces asociada a trombos hialinos en lúmenes capilares de los glomérulos.

La definición de substancia fibrinoide, desde el punto de vista histoquímico corresponde a aquella substancia que se presenta de color rojo anaranjado brillante con el Azan de Haidenhein, en partes iachuidas a veces en zonas mesangiales con metacromasia violeta o rojo azulada, o bien es rojo brillante con el tricrómico de Masson. Cuerpos hematoxilnicos no son considerados por nosotros de importancia en el diagnóstico histológico y suelen encontrarse en otras inflamaciones no relacionadas con el LES.

La definición exacta de nuestro material, dentro de lo que entendemos como enfermedad denominada LES con compromiso renal, está dada por: presencia de células LES circulantes, anticuerpos antinucleares circulantes y tisulares, inmunofluorescencia glomerular renal característica y estructuras supuestamente correspondientes a un polimixovirus en el glomérulo al microscopio electrónico. Los hechos anotados adquieren su verdadero valor al ser considerados en conjunto. Sin embargo si nos retrotraemos en el tiempo a numerosos y excelentes trabajos de numerosos autores, fundamentalmente clínicos, debemos aceptar sin duda que un buen número de casos tienen un aspecto cutáneo visceral bien definido $\mathrm{y}$ certero. Asimismo, las primeras y posteriores incursiones en la histología renal nos señalan aspectos que desde el punto de vista morfologico son inequívocos y por demás característicos en muchos casos con nefropatía.

De lo dicho se comprenderá mejor que la breve casuística presentada, sólo intenta dar a conocer un material seleccionado clínicamente, en el que en tres casos la histología ha contribuido en confirmar una lesión renal característica pero no específica, material por lo demás muy raro de observar entre adolecentes, siendo en ellos de muy mal pronóstico.

Discusión. Los hallazgos en cuanto a sexo y edad de nuestros pacientes coinciden con las experiencias de otros autores (3) (8) (9).

El compromiso renal citado como índice pronóstico en varias publicaciones (10) (11) (12) significó en nuestros enfermos un deterioro permanente y progresivo (casos 4,5 y 6 ), remisiones parciales (casos 3) y en el mejor de los casos estabilización de las lesiones (casos 1 y 2 ). Cabe destacar la enorme importancia de la realización de sedimentos urinarios en forma regular, ya que gracias a este predicamento nos fue posible pesquisar alteración renal antes de que se manifestara clínicamente (casos 1, 2, 5,). En tres de nuestros pacientes, este examen sirvió para confirmar la sos- 
pecha clínica (casos $3,4,6$,). La proteinuria y hematuria fueron los hallazgos constantes y mas significativos en nuestra pequeña casuística lo que concuerda con lo observado por otros autores (11) (10) (13).

El lapso entre comienzo de la colagenopatía y compromiso renal es variable, generalmente breve (11) (12). En dos casos esta complicación se presentó desde el comienzo (casos 5 y 6). Este lapso tiende a prolongarse con la edad del paciente (10). Cooks y cols. (9) agregan que los adultos responden en forma taás favorable a la terapia con corticoides.

Las características histológicas de Ta lesión renal son analizadas en el cuadro 5; sólo nos cabe insistir que en los casos 4 y 5 se aprecia una clara relación entre las graves manifestaciones clínicas y de laboratorio con la extensión del proceso en el glomérulo y el mal pronóstico. A la inversa los leves cambios histológicos en los pacientes 1 y 2 concuerdan con la buena respuesta terapéutica $y$ buen pronóstico inmediato.

Los dos pacientes que controlamos actualmente están con la dosis mínima de prednisona necesaria para controlar la enfermedad en base a niveles séricos de C3 (13). Diversos autores están de acuerdo en usar dosis tan altas de corticoides como los pacientes puedan tolerar (9) (10); Ackerman (14) nos da a conocer su experiencia con buenos resultados, al administrar corticoides a altas dosis y en días alternos. En dos de nuestros pacientes se usaron inmunosupresores: en uno a causa de los efectos secundarios de la prednisona (caso 3), y en otro (caso 1) una queratitis herpética nos obligó a suspender transitoriamente la terapia con esteroides.

\section{RESUMEN}

Se analizan seis casos de nefropatia lápica en la infancia, se destacan las manifestaciones proteiformes de la colagenopatía y la importancia del sedimento urinario en forma seriada para un diagnóstico precoz. Se analiza la histopatología renal en relación al pronóstico. Se señala criterio para el uso y dosis de corticoides.

\section{SUMMARY}

Six cases of LED with Nephropathy are considered. Their misleading clinical signs and the importance of frequence urinalysis are enphasized for early diagnosis. Five of these patients had a renal biopsy and its findings are correlated with prognosis. Criteria for treatment is considered.

\section{Bibliografía}

1.- Amas Cruz R.: El diagnóstico del lupus eritematoso. Rev. Méd. Chile 95: 628, 1967.
2.-Zweiman B, Komblum J., Cornag $Y$, and Hildreth E.: The prognosis of Lupus Nephritis. Ann Intern Med. 69: 441, 1968.

3.- Meislin AG, Rothfield N.: Systemic Lupus erythematosus in childhood. Pediatrics 43: 37, 1968.

4.-Good RA, Venters $H$., and Good TA: Diffuse connetive tissue in child bood, with special comment on connective tissue diseases in patiens with agammaglobulinemia. J. Lancet 81; 198, 1961.

5.- Kotfer D., Agnello V., Carr RI et al: Variable patterns of immunoglobulin and complement deposition in the kidneys of patiens with Systemic Lupus Erythematosus. Am J. Med. 56: 305, 1969.

6.- Ahmadian YS, Given GZ, and Mendoza SA: Normal urine and Positive immofluoressence reaction in Lupus Nephritis. Am J. Dis Child 123: 121, 1972.

7.-Cobhan AS, Reynolds WE, Franklin EC et al: Preliminary criteria for the classification of systemic Lupus Erytematosus. Bull Rbeum Dis 21: $643,1971$.

8.-Wallace S., Diamond H., and Kaplan D.: Recent Advances in Rheumatic Diseases 77: 455, 1972.

9.-Cook CD, Wedgwood RJP, Craig $J_{N}$, Hartman $J R$, and Janeway $C A$ : Systemic Lupus Erythematosus. Description of 37 cases in childhood and a discussion of endocrine Theraphy in 32 of the cases Pediatrics 26: 570, 1960.

10,-Wilson $R N$, Moher JF, and Shreiner GE: Lupus Nefhritis. Clinical and histological survery Arch Intern Med. 111: 424, 1963.

11.- Petersen RDA, Vernier RL. and Good RA.: Lupus Erythematosus Pediat. Clin. N. Am 10: 941, 1963.

12.-Soffer $L J$, Southren $A L$, Veiner RE. and Wolf $R L$.: Renal Manifestation of systemic Lupus Erythematosus: A clinical and pathological study of 90 cases Ann Intern Med. 54: 215, 1961.

13,- Pirani $C L$., and Mantaligod JR.: The kidney in Collagen Diseases. The kidney, ed. by Mostofl FK and Smith David E., The Williams \& Wilkins Company Baltimore, 1966.

14.-Ackerman G.: Alternate-Day Steroid Therapy in Lupus Nephritis Ann Intern Med, 72: 511, 1970.

15.-Pollak RV, Pirani LC., Schwartz FD.: The natural history of the renal manifestations of systemic Lupus Erythematosus. J. Lab. Clin. Med. 63: 537, 1964.

16.- Jalil JM., Armas Cruz R., Harnecker JJ. y González Felipe. Lupus eritematoso generalizado. Análisis de 100 casos tratados con corticoides SS. RR., y comparación con 25 casos tratados. Rev. Med. Chile, 717, 1958.

17.--Steinberg AD., Kaltraider HB, Staples Pj, et al: Cyclophosphamide in Lupus Nephritis, a controlled trial Ann Intern Med. 75: 165, 1971.

18.-Cameron JS., Boulton Jones M., Robinson R, et al: Treatment of Lupus Nefhritis whith cyclophosphamide Lancet 2: 846, 1970. 